\title{
Early Prognostic Factors Associated with the Efficacy of Infliximab Treatment for Patients with Rheumatoid Arthritis with Inadequate Response to Methotrexate
}

\author{
Satoko Hayashi · Katsuya Suzuki · Keiko Yoshimoto • Masaru Takeshita • \\ Takahiko Kurasawa · Kunihiro Yamaoka · Tsutomu Takeuchi
}

Received: October 16, 2015 / Published online: December 11, 2015

(C) The Author(s) 2015. This article is published with open access at Springerlink.com

\begin{abstract}
Introduction: Early prognostic factors for the clinical response in patients with rheumatoid arthritis (RA) after 1 year of treatment with infliximab (IFX) as part of routine clinical practice were investigated.

Methods: Thirty-five patients with RA with an inadequate response to methotrexate were enrolled and administered IFX (3-9 mg/kg, every $4-8$ weeks). Serum trough levels of IFX and levels of 9 cytokines were measured at baseline and at 3, 6 months, and 1 year. Associations between these parameters and clinical indicators were statistically analyzed.
\end{abstract}

Results: Serum trough levels of IFX and serum levels of interleukin (IL)- 6 in the early phase of IFX treatment were investigated. Patients with

Electronic supplementary material The online version of this article (doi:10.1007/s40744-015-0022-y) contains supplementary material, which is available to authorized users.

S. Hayashi · K. Suzuki - K. Yoshimoto $\cdot$ M. Takeshita T. Kurasawa $\cdot$ K. Yamaoka $\cdot$ T. Takeuchi $(\bowtie)$ Division of Rheumatology, Department of Internal Medicine, Keio University School of Medicine, Tokyo, Japan

e-mail: tsutake@z5.keio.jp low serum IL-6 achieved a higher clinical response as evaluated by the European League Against Rheumatism response criteria. Notably, the serum levels of IL- 6 and IL-10 at baseline exhibited a significant positive correlation with disease activity at 1 year. Low serum levels of IL-6 and IL-10 at baseline were associated with low Disease Activity Score 28 erythrocyte sedimentation rate (DAS28-ESR). Cut-off values of IL-6 $(5.45 \mathrm{pg} / \mathrm{mL})$ and IL-10 and $(1.68 \mathrm{pg} / \mathrm{mL})$ enabled discrimination of DAS28-ESR remission from non-remission with high sensitivity and moderate specificity.

Conclusion: Following the initiation of IFX treatment, early disease activity and remission were associated with serum levels of IL-6. Serum levels of IL-6 and IL-10 at baseline predict the efficacy after 1 year of treatment with IFX. Patients with high serum levels of IL-6 and IL-10 at baseline before IFX treatment might require more intensive therapy to achieve higher rates of clinical remission at 1 year.

Funding: Eisai Co., Ltd.

Keywords: Clinical efficacy; Infliximab; Interleukin-6; Prognostic serum marker; Rheumatoid arthritis 


\section{INTRODUCTION}

Rheumatoid arthritis (RA) is the most common and prototypical inflammatory arthropathy in adults. Tumor necrosis factor (TNF)- $\alpha$ plays an important role in the pathogenesis of RA and is considered a main therapeutic target of this disease [1, 2]. Infliximab (IFX) was the first chimeric anti-TNF- $\alpha$ antibody developed to treat RA and has demonstrated high efficacy in a number of studies, including the ATTRACT, BeSt, ASPIRE, and RECONFIRM studies [3-6].

The RISING study [7] and its subanalysis [8] intensively investigated factors associated with disease activity in patients with an inadequate response to methotrexate (MTX) after 1 year of treatment. This study divided patients into four groups using a combination of IFX serum trough level and interleukin (IL)-6 serum level at 1 year and found that the most favorable clinical response at this time was in the group with a high IFX serum trough level and low IL-6 serum level. These findings indicated that IFX's control of disease activity at 1 year depended on the degree of neutralization of not only TNF- $\alpha$ but also IL-6.

In addition, a subsequent subanalysis of the RISING study regarding the association of disease activity after 3 months of treatment with efficacy at 1 year [9] concluded that patients with lower activity at 3 months had a high probability of remission at 1 year. In that study, IFX dose and intervals were programmed and continued for 1 year as described in the study protocol. However, in clinical practice dose and interval are optimized for each patient using the treat-to-target (T2T) approach.

Serum TNF- $\alpha$ concentration at baseline is a prognostic factor for requirement of IFX administration [10, 11]. However, the optimal selection of biologics depends on a number of key factors, and optimum dose and interval for the control of disease activity with IFX treatment have not been established.

Here, we investigated early-phase clinical indicators and serum factors associated with the efficacy of IFX treatment for RA after treatment for 1 year in daily practice.

\section{METHODS}

\section{Patients}

Eligibility criteria for this study were a diagnosis of RA according to the 1987 revised criteria of the American College of Rheumatology (ACR) [12] or 2010 ACR/European League Against Rheumatism (EULAR) classification criteria [13]; initiation of IFX treatment between April 2010 and December 2012; inadequate response to MTX; and no previous treatment with biologics. All patients received intravenous administration of IFX (3-9 mg/kg, every 4-8 weeks) based on the domestic package insert under a T2T approach. No restriction was placed on the use of other concomitant treatments, including MTX and corticosteroids.

Observational and investigational study protocols were approved by the ethics committee of Keio University School of Medicine, Tokyo, Japan, and followed the Declaration of Helsinki and Good Clinical Practice guidelines. Written informed consent was obtained from all patients before investigation.

\section{Clinical Assessments}

Clinical assessments, including routine history, physical examination, and blood and urine tests were evaluated at baseline and immediately before IFX administration at 3, 6 months, and 
1 year. The following information was also collected: Disease Activity Score 28 erythrocyte sedimentation rate (DAS28-ESR), DAS28 C-reactive protein (DAS28-CRP), Simplified Disease Activity Index (SDAI), and Clinical Disease Activity Index (CDAI).

DAS28-ESR remission and clinical categories were defined as follows: remission, $<2.6$; low disease activity (LDA), 2.6 to $<3.2$; moderate disease activity (MDA), 3.2 to $<5.1$; and high disease activity (HDA), $\geq 5.1$. DAS28-CRP remission and clinical categories were defined as follows: remission, $<2.3$; LDA, 2.3 to $<2.7$; MDA, 2.7 to $\leq 4.1$; and HDA, $>4.1$. SDAI remission and clinical categories were defined as follows: remission, $<3.3$; LDA, 3.3 to $<11$; MDA, 11 to $\leq 26$; and HDA, $>26$. CDAI remission and clinical categories were defined as follows: remission, $<2.8$; LDA, 2.8 to $<10$; MDA, 10 to $\leq 22$; and HDA, $>22$.

Patients were classified into the following groups at 3 and 6 months: IFX-high/IL-6-high group, IFX-high/IL-6-low group, IFX-low/ IL-6-low group, and IFX-low/IL-6-high group. Cut-off values were based on the RISING study at $1.0 \mu \mathrm{g} / \mathrm{mL}$ for IFX serum trough level and $10 \mathrm{pg} / \mathrm{mL}$ for IL-6 serum level $[7,8]$.

\section{Measurement of Serum Trough Level of IFX and Serum Levels of Cytokines}

Peripheral blood was obtained at baseline and immediately before IFX administration at 3, 6 months, and 1 year. Serum was immediately separated by centrifugation and stored at $-80^{\circ} \mathrm{C}$ until use. IFX serum trough level was measured by enzyme-linked immunosorbent assay (ELISA; SIKARI QS-INFLIXI, Matriks Biotek, Ankara, Turkey) following the manufacturer's instructions. Serum concentrations of the following inflammatory cytokines were measured by ultra-sensitive electrochemiluminescence assay (Meso Scale Discovery, Rockville, MD, USA): granulocyte-macrophage colony-stimulating factor (GM-CSF), interferon (IFN)- $\gamma$, TNF- $\alpha$, and IL-1, $-2,-6,-8,-10$, and -12 . To minimize interference with immunoglobulin, including rheumatoid factor, an immunoglobulin-inhibiting reagent (Bioreclamation, New York, NY, USA) was applied during measurements. The limit of detection for IFX and each cytokine was as follows: IFX, (20 ng/mL), GM-CSF $(0.228 \mathrm{pg} /$ $\mathrm{mL}), \mathrm{IFN}-\gamma(0.453 \mathrm{pg} / \mathrm{mL}), \mathrm{IL}-10(0.214 \mathrm{pg} / \mathrm{mL})$, IL-12 (0.343 pg/mL), IL-1 (0.151 pg/mL), IL-2 (0.149 pg/mL), IL-6 (0.098 pg/mL), IL-8 $(0.062 \mathrm{pg} / \mathrm{mL})$, and TNF- $\alpha(0.134 \mathrm{pg} / \mathrm{mL})$.

\section{Statistical Analysis}

Continuous variables are shown as median and interquartile range (IQR) or percentage. Significant continuous variables were detected using Wilcoxon's test. Multiple groups were compared using Fisher's exact test. Patients were divided by IFX serum trough level and IL-6 serum level, and cut-off values were set according to the protocol of the RISING study $[7,8]$. To explore prognostic factors at baseline or early phase for clinical disease activity, univariate and multivariate analysis were applied. Variables were selected using a stepwise method. Cut-off values were determined using the receiver operating characteristics (ROC) curve. $P$ values $<0.05$ were considered significant. All analyses were conducted using JMP10 software, version 11.0 (SAS Institute Inc., Cary, NC, USA). 


\section{RESULTS}

\section{Patient Background}

Baseline characteristics of the 35 patients are shown in Table 1. Median (IQR) age was 53 (44-66) years and 29 (81\%) patients were

Table 1 Baseline characteristics of patients

\begin{tabular}{lc}
\hline Variables & \multicolumn{1}{l}{$\boldsymbol{N}=35$} \\
\hline Age (years) & $53(44-66)$ \\
Female, $n$ (\%) & $29(81)$ \\
Body weight $(\mathrm{kg})$ & $54.5(47-66)$ \\
Disease duration (years) & $2.41(0.75-7.5)$ \\
Methotrexate dose (mg/week) & $8(8-10)$ \\
Concomitant glucocorticoid, $n$ (\%) & $7(20)$ \\
DAS28-CRP & $4.17(3.07-5.82)$ \\
DAS28-ESR & $5.14(4.04-6.89)$ \\
SDAI & $22.8(15.0-42.2)$ \\
CDAI & $19.2(14.3-38.8)$ \\
Tender joint count (0-28) & $5(1-13)$ \\
Swollen joint count (0-28) & $7(4-12)$ \\
CRP (mg/dL) & $0.95(0.08-2.68)$ \\
ESR (mm/h) & $46(24-85)$ \\
Patients global VAS & $50(27-66)$ \\
Physicians global VAS & $50(18-75)$ \\
HAQ-DI score (unit) & $0.75(0.38-1.50)$ \\
RF (IU/mL) & $62(17-124)$ \\
ACPA (IU/mL) & $97.5(11.7-166.5)$ \\
MMP-3 (ng/mL) & $172.7(66.1-378.2)$ \\
\hline
\end{tabular}

Values are median and interquartile range unless otherwise stated

$A C P A$ anti-citrullinated peptide antibody, $C D A I$ Clinical Disease Activity Index, $C R P$ C-reactive protein, $D A S 28$ Disease Activity Score 28, ESR erythrocyte sedimentation rate, $H A Q-D I$ Health Assessment Questionnaire-Disability Index, $M M P$ - 3 matrix metalloproteinase-3, $R F$ rheumatoid factor, $S D A I$ Simplified Disease Activity Index, $V A S$ visual analogue scale female. Disease duration was $2.41(0.75-75)$ years. At baseline, disease activity assessed by DAS28-ESR was high (5.14 median), but dose of concomitant MTX was $8 \mathrm{mg} /$ week (median). In three patients, IFX administration was discontinued after 7 months due to lack of efficacy, economical reasons, or adverse events (infusion reaction). Analysis at 1 year for 3 patients was done using the last observation carried forward method.

\section{Changes in Clinical Response and Serum Trough Level of IFX and Serum Levels of Cytokines}

DAS28-ESR was 5.15 at baseline and was significantly reduced to 2.82 at 3 months, 2.68 at 6 months, and 2.49 at 1 year $(P<0.0001$; Table 2). Median CDAI was 19.2 at baseline and significantly reduced to 5.1 at 3 months, 3.3 at 6 months, and 1.95 at 1 year $(P<0.0001$; Table 2). Serum matrix metalloproteinase-3 (MMP-3) levels and DAS28-CRP were also significantly reduced after 3 months. Chronological changes in representative values are shown in Figure $S 1$ in the supplementary material.

Serum trough level of IFX was $2.92 \mu \mathrm{g} / \mathrm{mL}$ (median) at 3 months, which exceeds the $1 \mu \mathrm{g}$ / $\mathrm{mL}$ considered as the minimally effective concentration. This concentration was maintained until 1 year. The percentage of patients with serum IFX concentration of more than $1 \mu \mathrm{g} / \mathrm{mL}$ was over $70 \%$ of all patients. Serum levels of GM-CSF, IFN- $\gamma$, IL-12, and IL-6 were significantly reduced at 1 year compared to those at baseline. Remarkably, IL-6 was dramatically reduced from $4.29 \mathrm{pg} / \mathrm{ml}$ at baseline to $0.36 \mathrm{pg} / \mathrm{mL}$ at 1 year $(P<0.0001)$. 


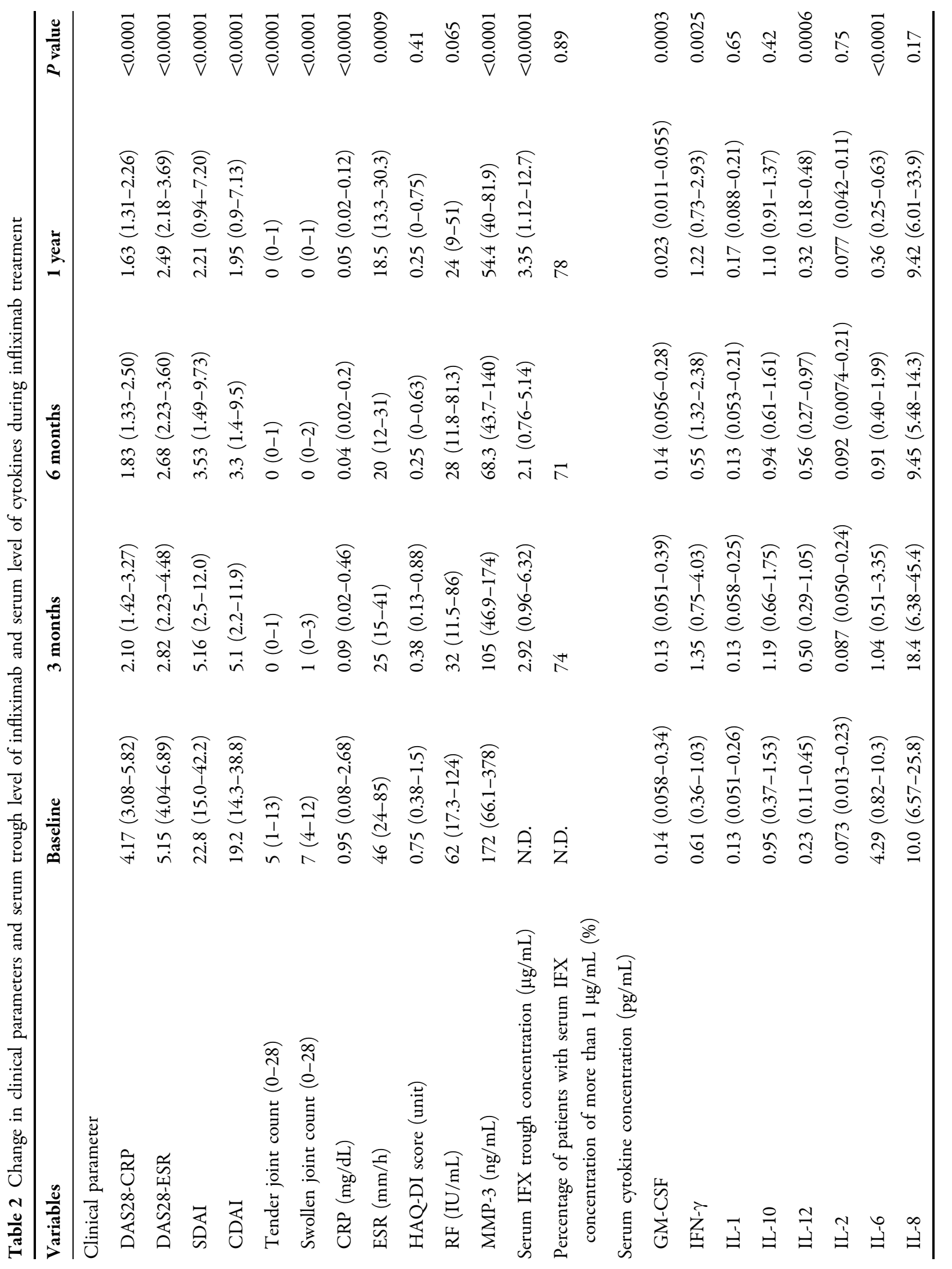




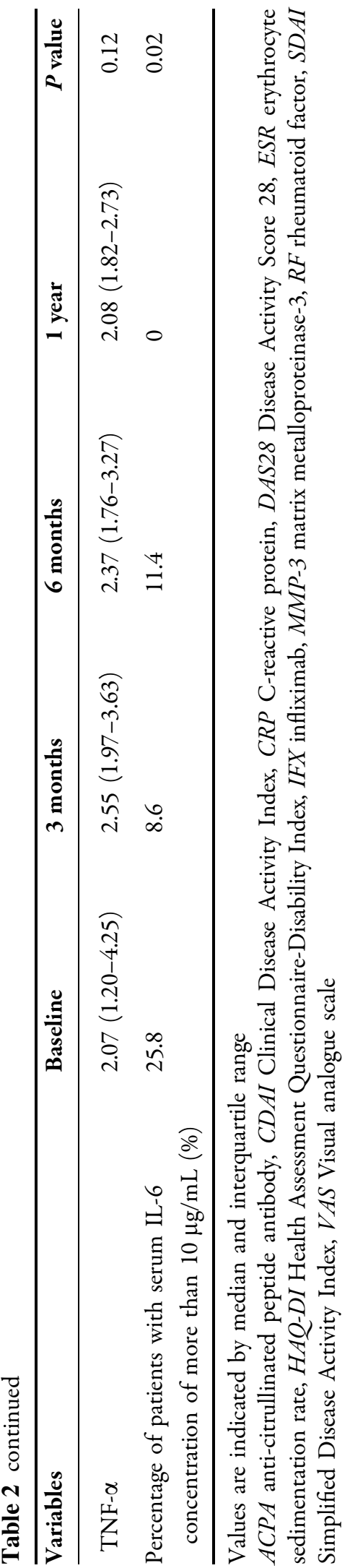

\section{Association Among Serum Trough Levels of IFX, Serum Levels of Cytokines, and Clinical Responses in the Early Phase of IFX Treatment}

Comparison of disease activity among the four groups showed significant differences at both 3 and 6 months. Namely, disease activity was lower in the IFX-high/IL-6-low and IFX-low/ IL-6-low groups. In addition, serum CRP levels were lower in these groups. However, in contrast to the RISING study serum levels of rheumatoid factor, anti-citrullinated peptide antibody, and MMP-3 did not differ between groups $[7,8]$.

To confirm a direct association between the IFX serum trough level and clinical response, patients were divided using the same cut-off values into IFX-high and -low groups and disease activity was compared at 3 and 6 months. Disease activity tended to be lower in the IFX-high group at both 3 and 6 months. These results suggest that serum IL-6 level, rather than IFX serum trough level, potentially reflects TNF- $\alpha$ inhibition and disease activity.

Further, prediction of disease activity at 1 year was attempted using the classification model, a combination IFX serum trough level and IL- 6 serum level at 3 and 6 months. However, no significant association was observed.

\section{Association Between Serum Cytokine Levels at Baseline and Clinical Response at 1 Year}

To identify baseline factors associated with efficacy at 1 year, correlations between clinical parameters and serum cytokine levels at baseline to disease activity at 1 year were compared by univariate analysis. Although clinical parameters at baseline were not 
significantly correlated with disease activity, serum levels of IL-6 and IL-10 were significantly correlated, as shown in Table 3.

To determine independent variables for prognosis, we then conducted a multivariate analysis. Notably, IL- 6 and IL-10 were associated with disease activity as evaluated by DAS28-ESR at 1 year (Table 4). When CDAI was used for evaluation, IL-2, -6, and -10 were associated with disease activity. In categorical classifications, IL-6 and IL-10 were associated with remission as determined by DAS28-ESR and CDAI. Cut-off values of 5.45 and $5.46 \mathrm{pg} /$ $\mathrm{mL}$ for IL-6 and 1.68 and $2.36 \mathrm{pg} / \mathrm{mL}$ for IL-10 discriminated remission from non-remission by DAS28-ESR or CDAI at 1 year. The sensitivity and specificity of evaluation by DAS28-ESR was 0.80 and 0.67 for IL- 6 and 0.95 and 0.5 for IL-10 (Fig. 1). The sensitivity and specificity of evaluation by CDAI was 0.80 and 0.67 for IL- 6 and 1.0, 0.33 for IL-10. Values of the area under the curve were 0.76 for IL- 6 and 0.69 for IL-10, indicating moderate accuracy.

\section{DISCUSSION}

In this single-center, observational and investigational cohort study, we demonstrated that IL-6 serum level rather than IFX serum trough level was associated with disease activity at 3 and 6 months after the initiation of IFX treatment. In addition, serum levels of IL- 6 and IL-10 at baseline were significantly associated with efficacy after 1 year of treatment with IFX for RA patients with an inadequate response to MTX.

Several studies have analyzed the kinetics of serum inflammatory cytokines following IFX administration [7, 8, 14-17]. In some of these studies, serum levels of IL-6 decreased in response to IFX administration [14-16]. Fourteen weeks after the initiation of IFX
Table 3 Univariate analysis of associations between baseline clinical parameters and serum cytokines and clinical remission at 1 year

\begin{tabular}{|c|c|c|}
\hline \multirow[t]{2}{*}{ Baseline variables } & \multicolumn{2}{|c|}{ Clinical remission at 1 year } \\
\hline & $\begin{array}{l}\text { DAS28-ESR } \\
(P \text { value })\end{array}$ & $\begin{array}{l}\text { CDAI } \\
(P \text { value })\end{array}$ \\
\hline \multicolumn{3}{|l|}{ Clinical parameters } \\
\hline Age & 0.74 & 0.96 \\
\hline Disease duration & 0.45 & 0.50 \\
\hline DAS28-CRP & 0.22 & 0.23 \\
\hline DAS28-ESR & 0.08 & 0.14 \\
\hline SDAI & 0.27 & 0.37 \\
\hline CDAI & 0.23 & 0.34 \\
\hline $\begin{array}{l}\text { Tender joint count } \\
(0-28)\end{array}$ & 0.15 & 0.12 \\
\hline $\begin{array}{l}\text { Swollen joint count } \\
(0-28)\end{array}$ & 0.85 & 0.89 \\
\hline CRP & 0.80 & 0.96 \\
\hline ESR & 0.42 & 0.63 \\
\hline HAQ-DI score & 0.41 & 0.63 \\
\hline RF & 0.47 & 0.98 \\
\hline ACPA & 0.50 & 0.50 \\
\hline MMP-3 & 0.60 & 0.91 \\
\hline \multicolumn{3}{|c|}{ Serum cytokine concentration } \\
\hline GM-CSF & 0.33 & 0.28 \\
\hline IFN- $\gamma$ & 0.60 & 0.17 \\
\hline IL-1 & 0.80 & 0.95 \\
\hline IL-10 & 0.017 & 0.031 \\
\hline IL-12 & 0.22 & 0.33 \\
\hline IL-2 & 0.17 & 0.10 \\
\hline IL-6 & 0.030 & 0.030 \\
\hline IL-8 & 0.052 & 0.053 \\
\hline TNF- $\alpha$ & 0.040 & 0.12 \\
\hline
\end{tabular}

$P$ values are indicated

$A C P A$ anti-citrullinated peptide antibody, $C D A I$ Clinical Disease Activity Index, CRP C-reactive protein, DAS28 Disease Activity Score 28, ESR erythrocyte sedimentation rate, $\quad G M-C S F \quad$ granulocyte-macrophage colony-stimulating factor, $H A Q-D I$ Health Assessment Questionnaire-Disability Disability Index, IFN interferon, $I L$ interleukin, $M M P-3$ matrix metalloproteinase-3, $R F$ rheumatoid factor, SDAI Simplified Disease Activity Index, $T N F$ tumor necrosis factor, $V A S$ visual analogue scale 
Table 4 Multivariate analysis of the association of extracted baseline serum cytokines with clinical remission at 1 year

\begin{tabular}{lll}
\hline Variables & OR $(\mathbf{9 5 \%}$ CI $)$ & $P$ value \\
\hline Clinical remission at 1 year & \\
DAS28-ESR & & \\
IL-10 & $1.92(1.04-7.43)$ & 0.015 \\
IL-6 & $1.09(1.01-1.26)$ & 0.0026 \\
CDAI at week 52 & & \\
IL-10 & $1.46(1.02-4.46)$ & 0.022 \\
IL-2 & $41.8(0.37-8588)$ & 0.12 \\
IL-6 & $1.08(1.01-1.25)$ & 0.025 \\
\hline
\end{tabular}

Calculated $P$ values, $\mathrm{OR}$, and $95 \% \mathrm{CIs}$ are indicated $C D A I$ Clinical Disease Activity Index, $C I$ confidence interval, DAS28 Disease Activity Score 28, ESR erythrocyte sedimentation rate, $I L$ interleukin, $O R$ odds ratio

administration, a decrease in the serum concentration was observed for IL-6, but not for TNF- $\alpha[14,15]$. Regarding reports of earlier time points following the initiation of IFX administration, the serum level of IL- 6 was immediately reduced and maintained, whereas that of TNF- $\alpha$ was temporarily increased and recovered to baseline level [16]. In the current study, a significant decrease in the serum level of IL-6 at 3 months compared to baseline and a trend for a further reduction at 6 months and 1 year was observed. However, the serum concentration of TNF- $\alpha$ did not change during the 1-year period, which is consistent with the reports above. In addition, when using the optimized administration method based on the T2T approach, the dose and intervals of IFX administration were not constant and serum levels of IL-6 significantly decreased over the 1-year period. This sequential observational study over 1 year to be is therefore considered to be unique and to give considerable weight to routine clinical practice.
Few reports have described serum factors prognostic for the efficacy of IFX. One reported prognostic factor is baseline serum concentration of TNF- $\alpha[7,8]$. Another report indicated that IL-1 $\beta$ concentration in whole blood stimulated by lipopolysaccharide was prognostic of efficacy at 6 months after the initiation of IFX treatment [17]. The current study clearly demonstrates that baseline serum concentrations of both IL-6 and IL-10 are prognostic for the efficacy of IFX treatment at 1 year.

IL-6 is a proinflammatory cytokine which is strongly associated with the pathophysiology of RA. In contrast, IL-10 is considered a pleiotropic cytokine with suppressive functions, and its role in RA is controversial. The serum concentration of IL-10 was reportedly not affected by 1 month of treatment with IFX [7]. Increases in the serum concentration of IL-10 were observed in patients with RA compared to healthy controls [18]. In addition, IL-10-deficient animal models manifest arthritis-exacerbated bone erosion [19]. Further, TNF- $\alpha$ inhibition was reported to induce IL-10 production from $\mathrm{CD} 4^{+} \mathrm{T}$ cells [20]. Therefore, the role of IL-10 in the pathophysiology of RA remains controversial. The results of the current study show that low baseline IL-10 concentration association with favorable outcome may indicate that IL-10 can associate with response to TNF inhibition under abnormal cytokine condition in RA. And it requires further study of molecular mechanisms to clarify association between serum levels of IL-10 and TNF- $\alpha$ inhibition.

However, a number of limitations also warrant mention. First, in the study design, the sample size was relatively small. Although reliable clinical data with relatively low bias were collected by experienced rheumatologists at a high-volume single center, sensitive techniques, including an ultra-sensitive assay 
Fig. 1 Cut-off values of baseline serum levels of IL-6 and IL-10 for the prediction of clinical remission at 1 year. Cut-off values of baseline serum IL-6 and IL-10 concentration were $A U C$ area under curve, IL interleukin, $R O C$ receiver operating characteristics calculated by ROC.
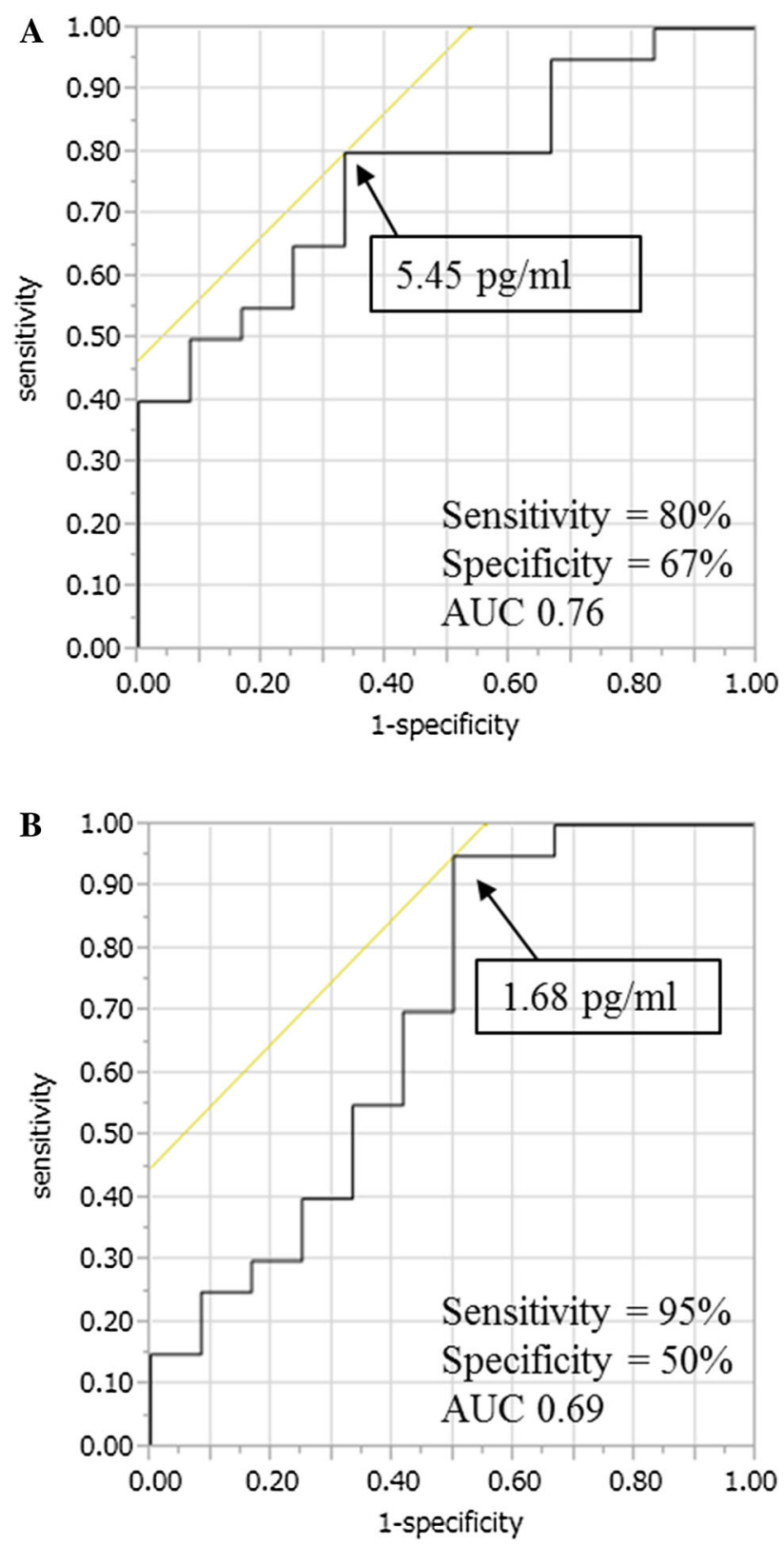

for the detection of extremely low concentrations of cytokines in serum were used, and variables such as cytokines in a cohort comprehensively and chronologically measured, data may be required external validation using different data set. Second, administration based on the T2T approach in the clinical setting of IFX serum trough level and serum cytokine level might be influenced by IFX interval and dose, as well as concomitant drugs. In this regard, the serum trough level of IFX might not be an optimal prognostic marker when using the T2T approach. The data and previous insights were therefore interpreted with care and it was determined that the serum concentration of IL-6 is the most stable and reliable prognostic factor at baseline and the most stable and reliable disease activity 
marker at 3 and 6 months of IFX treatment. Further study of IFX is required to alleviate these concerns.

\section{CONCLUSIONS}

In conclusion, disease activity and remission in the early phase of IFX therapy are associated with the serum level of IL-6. Serum levels of IL- 6 and IL-10 at baseline predicted the efficacy of IFX at 1 year. Patients with an inadequate response to MTX and a high serum level of IL- 6 or IL-10 at baseline might be refractory to IFX treatment. Patients with high serum levels of IL-6 in the early phase of IFX treatment may require more intensive treatment at these time points or shorter administration intervals to achieve higher levels of clinical remission at 1 year.

\section{ACKNOWLEDGMENTS}

This study was funded by Eisai Co., Ltd. and by internal research grants from Keio University. The authors thank the patients and staff involved in this study. The authors also thank Dr. Guy Harris (DMC Corp.) for editing of the manuscript. Support for this assistance was funded by Eisai Co., Ltd. All named authors meet the International Committee of Medical Journal Editors (ICMJE) criteria for authorship for this manuscript, take responsibility for the integrity of the work as a whole, and have given final approval for the version to be published.

Disclosures. KS has received research grants from Eisai Co., Ltd. and Bristol-Myers Squibb. TT has received grants from Astellas Pharma; Bristol-Myers K.K.; Chugai Pharmaceutical Co.,
Ltd.; Daiichi Sankyo Co., Ltd.; Eisai Co., Ltd.; Mitsubishi Tanabe Pharma Co.; Pfizer Japan Inc.; Santen Pharmaceutical Co., Ltd.; Takeda Pharmaceutical Co., Ltd.; Teijin Pharma Ltd.; AbbVie G.K.; Asahikasei Pharma Corp.; Taisho Toyama Pharmaceutical Co., Ltd.; and SymBio Pharmaceuticals Ltd.; speaking fees from AbbVie G.K., Bristol-Myers K.K.; Chugai Pharmaceutical Co,. Ltd.; Eisai Co., Ltd.; Janssen Pharmaceutical K.K.; Mitsubishi Tanabe Pharma Co.; Pfizer Japan Inc.; Takeda Pharmaceutical Co., Ltd.; Astellas Pharma; Diaichi Sankyo Co., Ltd.; Celtrion; and Nipponkayaku Co., Ltd.; and consultant fees from Astra Zeneca K.K., Eli Lilly Japan K.K., Novartis Pharma K.K., Mitsubishi Tanabe Pharma Co.; Asahi Kasei Medical K.K.; AbbVie G.K.; Daiichi Sankyo Co., Ltd.; Bristol-Myers K.K.; and Nipponkayaku Co., Ltd.

\section{Compliance with Ethics Guidelines.} Observational and investigational study protocols were approved by the ethics committee of Keio University School of Medicine, Tokyo, Japan, and followed the Declaration of Helsinki and Good Clinical Practice guidelines. Written informed consent was obtained from all patients before investigation.

Open Access. This article is distributed under the terms of the Creative Commons Attribution-NonCommercial 4.0 International License (http://creativecommons.org/licenses/ by-nc/4.0/), which permits any noncommercial use, distribution, and reproduction in any medium, provided you give appropriate credit to the original author(s) and the source, provide a link to the Creative Commons license, and indicate if changes were made. 


\section{REFERENCES}

1. Elliott MJ, Maini RN, Feldmann M, et al. Treatment of rheumatoid arthritis with chimeric monoclonal antibodies to tumor necrosis factor alpha. Arthritis Rheum. 1993;36:1681-90.

2. Tetta C, Camussi G, Modena V, Di Vittorio C, Baglioni C. Tumour necrosis factor in serum and synovial fluid of patients with active and severe rheumatoid arthritis. Ann Rheum Dis. 1990;49:665-7.

3. Smolen JS, Han C, Bala M, et al. ATTRACT Study Group. Evidence of radiographic benefit of treatment with infliximab plus methotrexate in rheumatoid arthritis patients who had no clinical improvement: a detailed subanalysis of data from the anti-tumor necrosis factor trial in rheumatoid arthritis with concomitant therapy study. Arthritis Rheum. 2005;52:1020-30.

4. Goekoop-Ruiterman YP, de Vries-Bouwstra JK, Allaart CF, et al. Clinical and radiographic outcomes of four different treatment strategies in patients with early rheumatoid arthritis (the BeSt study): a randomized, controlled trial. Arthritis Rheum. 2008;58:S126-35.

5. Smolen JS, Han C, van der Heijde DM, et al. Active-Controlled Study of Patients Receiving Infliximab for the Treatment of Rheumatoid Arthritis of Early Onset (ASPIRE) Study Group. Radiographic changes in rheumatoid arthritis patients attaining different disease activity states with methotrexate monotherapy and infliximab plus methotrexate: the impacts of remission and tumor necrosis factor blockade. Ann Rheum Dis. 2009;68:823-7.

6. Yamanaka H, Tanaka Y, Sekiguchi $\mathrm{N}$, et al. Retrospective clinical study on the notable efficacy and related factors of infliximab therapy in a rheumatoid arthritis management group in Japan (RECONFIRM). Mod Rheumatol. 2007;17:28-32.

7. Takeuchi $\mathrm{T}$, Miyasaka N, Inoue $\mathrm{K}$, Abe $\mathrm{T}$, Koike $\mathrm{T}$. RISING study. Impact of trough serum level on radiographic and clinical response to infliximab plus methotrexate in patients with rheumatoid arthritis: results from the RISING study. Mod Rheumatol. 2009;19:478-87.

8. Takeuchi T, Miyasaka N, Tatsuki Y, et al. Inhibition of plasma IL- 6 in addition to maintenance of an efficacious trough level of infliximab associated with clinical remission in patients with rheumatoid arthritis: analysis of the RISING Study. Ann Rheum Dis. 2012;71:1583-5.

9. Takeuchi T, Miyasaka N, Inui T, et al. Prediction of clinical response after 1 year of infliximab therapy in rheumatoid arthritis based on disease activity at 3 months: post hoc analysis of the RISING study. J Rheumatol. 2015;42(4):599-607 [Erratum (2015). J Rheumatol;42(5):905, check].

10. Edrees AF, Misra SN, Abdou NI. Anti-tumor necrosis factor (TNF) therapy in rheumatoid arthritis: correlation of TNF-alpha serum level with clinical response and benefit from changing dose or frequency of infliximab infusions. Clin Exp Rheumatol. 2005;23:469-74.

11. Takeuchi T, Miyasaka N, Tatsuki Y, et al. Baseline tumour necrosis factor alpha levels predict the necessity for dose escalation of infliximab therapy in patients with rheumatoid arthritis. Ann Rheum Dis. 2011;70:1208-15.

12. Arnett FC, Edworthy SM, Bloch DA, et al. The American Rheumatism Association 1987 revised criteria for the classification of rheumatoid arthritis. Arthritis Rheum. 1988;31:315-24.

13. Aletaha D, Neogi T, Silman AJ, et al. Rheumatoid arthritis classification criteria: an American College of Rheumatology/European League Against Rheumatism collaborative initiative. Arthritis Rheum. 2010;62:2569-81.

14. Ingegnoli F, Fantini F, Favalli EG, et al. Inflammatory and prothrombotic biomarkers in patients with rheumatoid arthritis: effects of tumor necrosis factor-alpha blockade. J Autoimmun. 2008;31:175-9.

15. Schulz M, Dotzlaw H, Neeck G. Ankylosing spondylitis and rheumatoid arthritis: serum levels of TNF- $\alpha$ and its soluble receptors during the course of therapy with etanercept and infliximab. Biomed Res Int. 2014;2014:675108. doi:10.1155/2014/ 675108

16. Charles P, Elliott MJ, Davis D, et al. Regulation of cytokines, cytokine inhibitors, and acute-phase proteins following anti-TNF-alpha therapy in rheumatoid arthritis. J Immunol. 1999;163:1521-8.

17. Kayakabe K, Kuroiwa $\mathrm{T}$, Sakurai $\mathrm{N}$, et al. Interleukin-1 $\beta$ measurement in stimulated whole blood cultures is useful to predict response to anti-TNF therapies in rheumatoid arthritis. Rheumatology (Oxford). 2012;51:1639-43. 
18. Shrivastava AK, Singh HV, Raizada A. Inflammatory markers in patients with rheumatoid arthritis. Allergol Immunopathol (Madr). 2015;43:81-7.

19. Greenhill CJ, Jones GW, Nowell MA, et al. Interleukin-10 regulates the inflammasome-driven augmentation of inflammatory arthritis and joint destruction. Arthritis Res Ther. 2014;16:419.

20. Evans HG, Roostalu U, Walter GJ, et al. TNF- $\alpha$ blockade induces IL-10 expression in human CD4+ T cells. Nat Commun. 2014;5:3199. 